\title{
Linear Approach for Synchronous State Stability in Fully Connected PLL Networks
}

\author{
José R. C. Piqueira, ${ }^{1}$ Maurizio Q. de Oliveira, ${ }^{1}$ and Luiz H. A. Monteiro ${ }^{1,2}$ \\ ${ }^{1}$ Escola Politécnica, Universidade de São Paulo, Avenida Professor Luciano Gualberto, Travessa 3, \\ no. 158, 05508-900 São Paulo, SP, Brazil \\ ${ }^{2}$ Escola de Engenharia, Universidade Presbiteriana Mackenzie, Rua da Consolacao, 896 São Paulo, \\ SP 01302-907, Brazil
}

Correspondence should be addressed to José R. C. Piqueira, piqueira@lac.usp.br

Received 10 October 2007; Accepted 7 March 2008

Recommended by Jerzy Warminski

\begin{abstract}
Synchronization is an essential feature for the use of digital systems in telecommunication networks, integrated circuits, and manufacturing automation. Formerly, master-slave (MS) architectures, with precise master clock generators sending signals to phase-locked loops (PLLs) working as slave oscillators, were considered the best solution. Nowadays, the development of wireless networks with dynamical connectivity and the increase of the size and the operation frequency of integrated circuits suggest that the distribution of clock signals could be more efficient if distributed solutions with fully connected oscillators are used. Here, fully connected networks with second-order PLLs as nodes are considered. In previous work, how the synchronous state frequency for this type of network depends on the node parameters and delays was studied and an expression for the long-term frequency was derived (Piqueira, 2006). Here, by taking the first term of the Taylor series expansion for the dynamical system description, it is shown that for a generic network with $N$ nodes, the synchronous state is locally asymptotically stable.
\end{abstract}

Copyright (C) 2008 José R. C. Piqueira et al. This is an open access article distributed under the Creative Commons Attribution License, which permits unrestricted use, distribution, and reproduction in any medium, provided the original work is properly cited.

\section{Introduction}

Digital engineering technologies for communications, control, and computation require reliable clock distribution systems to guarantee the correct temporal order in the information processing by the several parts of a spatially distributed system [1-4]. Synchronization network is the general denomination of the part of the whole system responsible for this temporal order and the several possible solutions for its design are presented in [5].

Originally, master-slave architectures were used to distribute a precise clock signal generated by a master node to the other points of the systems where PLLs regenerate the phase and frequency information $[1,5]$. 
The evolution of the telecommunication services to wireless and dynamical networks hasshown the inadequacy of centralized clock distribution structures in these cases, motivating the study of fully connected architectures to generate reference signals with the phase-locked loops operating as nodes of the synchronization networks [1,5-7].

Besides telecommunication networks, the main fields for the application of fully connected systems are time signal distribution in digital electronic circuits [2, 7-9] and wireless sensor networks [10]. Another very important application of networks is the implementation of oscillatory neural-computing devices, where the vector of phase-differences amongst a group of synchronized oscillators is associated with some memory information [11, 12].

In this work, a fully connected $N$-node PLL network is studied, starting with a review of the model for a single node and the derivation of the synchronous state frequency [13]. Then, the dynamical equation for the phase differences between nodes is presented providing a model for the whole $N$-node network.

As it was shown in $[13,14]$, this kind of network presents a synchronous state that is reachable for any possible combination of node parameters. Consequently, the linear approximation gives practical hints about the lock-in operation of the network [15]. Then, considering the first term of the Taylor series development around the synchronous state [16], it is shown that the synchronous state is locally asymptotically stable for any number $N$ of nodes.

\section{Nodes in a fully connected architecture and synchronous state frequency}

In this section, the nodes of the fully connected network are analyzed, considering that they are second-order phase-locked loops modeled as the classical analog version [17]. The results shown in [13] are summarized and the expression of synchronous state frequency is derived. This expression will be used to determine the stability conditions. PLL nodes are closed loops composed of a phase detector (PD), a low-pass filter (F), and a voltage-controlled oscillator (VCO) [17]. The double frequency jitter in the output of the phase detector is neglected [1820].

The $N$ nodes are geographically distributed and mutually coupled by using two-way connections. Each $i$-node exchanges clock signals with all the $j$-nodes such that $j \neq i$. Each VCO belonging to a PLL is described by its free-running frequency $\omega_{i}$ and by its phase $\phi_{i}(t)$. The whole network has its dynamics described by phase errors and frequency errors defined by $\Delta \phi_{j i}(t)$ and $\Delta \dot{\phi}_{j i}(t)$, respectively. Time delays $\tau_{j i}$ corresponding to the propagation of signals from the node $j$ VCO output to the node $i$ PD input are considered, as shown in Figure 1, containing the model of the signal processed in node 1.

It can be noticed that in an $N$-node fully connected PLL network, the nodes need $N-1$ phase detectors [21] where the local VCO output signal is multiplied by the delayed signals sent by the VCO of the other nodes. As there is no integrated circuit with this architecture, the implementation of the nodes requires a combination of several PLL chips with the outputs of their phase detectors weighted and the result being the input of a single filter that feeds a single VCO. For the node 1 from Figure 1, the definitions are as follows:

(i) $\tau_{21}$ : delay from node 2 to node 1 ;

(ii) $\tau_{31}$ : delay from node 3 to node 1 ;

(iii) $\tau_{N 1}$ : delay from node $N$ to node 1 ;

(iv) $v_{1}(t)$ : VCO output of the node 1 ; 


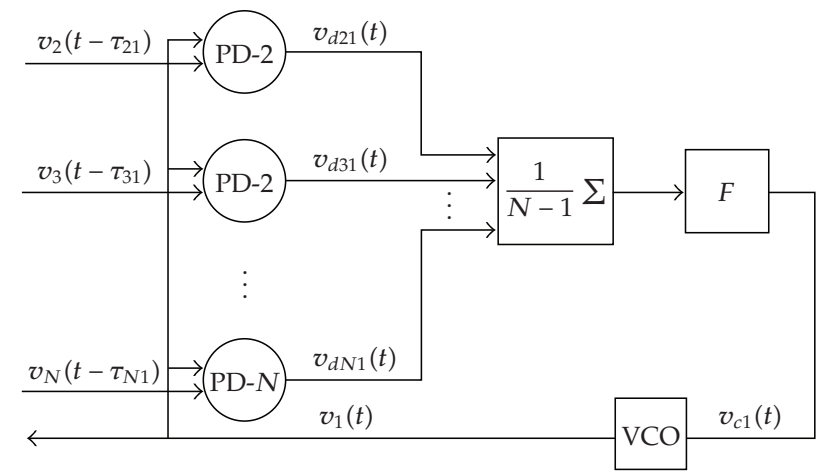

Figure 1: Node 1 signal processing in a fully connected PLL network.

(v) $v_{2}\left(t-\tau_{21}\right)$ : VCO output of the node 2 with a delay $\tau_{21}$;

(vi) $v_{3}\left(t-\tau_{31}\right)$ : VCO output of the node 3 with a delay $\tau_{31}$;

(vii) $v_{N}\left(t-\tau_{N 1}\right)$ : VCO output of the node $N$ with a delay $\tau_{N 1}$;

(viii) $v_{d 21}(t)$ : output of PD 2;

(ix) $v_{d 31}(t)$ : output of PD 3;

(x) $v_{d N 1}(t)$ : output of PD N;

(xi) $v_{c 1}(t)$ : output of $\mathrm{F}$ that controls the VCO.

The instantaneous individual VCO phases and the instantaneous phase errors, $\phi_{i}(t)$ and $\Delta \phi_{j i}(t)$, are expressed in the forms

$$
\begin{gathered}
\phi_{i}(t)=\omega_{i} t+\theta_{i}(t), \\
\Delta \phi_{j i}(t)=\phi_{j}(t)-\phi_{i}(t) .
\end{gathered}
$$

The node parameters related to PLL operation can be characterized by the following:

(i) PD multiplying factors: $k_{m_{1}}=k_{m_{2}}=\cdots=k_{m_{i}}=k_{m}$, in volts ${ }^{-1}$, with $i=1,2, \ldots, N$;

(ii) gains of the VCOs: $k_{1}=k_{2}=\cdots=k_{i}=k_{0}$, in $\mathrm{rad} / \mathrm{sV}$, with $i=1,2, \ldots, N$;

(iii) cut-off frequencies of F: $\mu_{1_{1}}=\mu_{1_{2}}=\mu_{1_{i}}=\cdots=\mu_{1}$, in rad/s, with $i=1,2, \ldots, N$.

The constitutive parameters of the nodes are considered to be the same, in order to simplify the analytical reasoning. If the filters cut-off frequencies are different, but sufficient to avoid double frequency jitter [18], the results derived here are basically the same and only the acquisition times change [13]. If VCO and PD gains are considered to be different, the problem has to be numerically treated, and the expression of the synchronous state frequency derived here changes [22].

Under the assumption that all nodes have the same constitutive parameters, the output of each VCO is

$$
v_{i}(t)=V \cos \phi_{i}(t) ;
$$


and signals received by the phase detector of node $i$ from node $j$, with propagation delays $\tau_{j i}$, can be written as

$$
v_{j}\left(t-\tau_{j i}\right)=V \sin \left[\phi_{j}\left(t-\tau_{j i}\right)\right],
$$

where $V$ is the controlled amplitude of the outputs of VCOs and PDs. Considering that each phase detector $j \neq i$, belonging to node $i$, is a signal multiplier [17]:

$$
v_{d_{j i}}(t)=k_{m} v_{j}\left(t-\tau_{j i}\right) v_{i}(t)
$$

replacing (2.2) and (2.3) in (2.4), and neglecting the double frequency components [18-20] as their frequency are much greater than the filter cut-off frequency, the output of each phase detector $j \neq i$, belonging to node $i$, is given by

$$
v_{d_{j i}}(t)=k_{m} V^{2}\left[\sin \phi_{j}\left(t-\tau_{j i}\right) \cos \phi_{i}(t)\right] .
$$

Each resulting signal given by $(2.5)$ is multiplied by $1 /(N-1)$ and added, in order to compose the filter input as below:

$$
v_{d_{i}}(t)=\left(\frac{1}{N-1}\right)\left(\frac{k_{m} V^{2}}{2}\right)\left\{\sum_{j=1, j \neq i}^{N} \sin \left[\phi_{j}\left(t-\tau_{j i}\right)-\phi_{i}(t)\right]\right\}
$$

Defining $k_{d}=(1 / 2)\left(k_{m} V^{2}\right)$, expression (2.6) is simplified to

$$
v_{d_{i}}(t)=\frac{k_{d}}{N-1}\left\{\sum_{j=1, j \neq i}^{N} \sin \left[\phi_{j}\left(t-\tau_{j i}\right)-\phi_{i}(t)\right]\right\} .
$$

The filters are considered to be first-order low-pass implying second-order nodes. This choice is a common practice because second-order PLLs always reach a synchronous state when submitted to phase steps and ramps [17] in spite of the double-frequency jitter [19,20]. If more accurate transient responses are necessary, second-order filters are used, but complicated behaviors like bifurcation and chaos appear [23], worsening the operation.

Consequently, equations for the dynamics of the VCO phase are obtained by considering the filter transfer function $F_{i}(s)=\mu_{1} /\left(s+\mu_{1}\right)$ [15], resulting in the expression

$$
\dot{v}_{c_{i}}(t)+\mu_{1} v_{c_{i}}(t)=\mu_{1} v_{d_{i}}(t)
$$

Replacing $v_{d_{i}}$, given by (2.7), and the VCO control signal $v_{c_{i}}=\dot{\theta}_{i}(t) / k_{0}$ in (2.8), the equation for the node phase is

$$
\ddot{\theta}_{i}(t)+\mu_{1} \dot{\theta}_{i}(t)-\mu_{1}\left(\frac{k_{o} k_{d}}{(N-1)}\right)\left\{\sum_{j=1, j \neq i}^{N} \sin \left[\phi_{j}\left(t-\tau_{j i}\right)-\phi_{i}(t)\right]\right\}=0 .
$$


Then, considering $\phi_{i}(t)=w_{i} t+\theta_{i}(t)$ in (2.9) and defining $\mu_{2}=k_{o} k_{d}$ and $k=\mu_{1} \mu_{2} /(N-1)$, the dynamics of each VCO phase in a fully connected network is given by

$$
\ddot{\phi}_{i}(t)+\mu_{1} \dot{\phi}_{i}(t)-\mu_{1} \omega_{i}-k\left\{\sum_{j=1, j \neq i}^{N} \sin \left[\phi_{j}\left(t-\tau_{j i}\right)-\phi_{i}(t)\right]\right\}=0 .
$$

Equation (2.10) is similar to the pendulum equation, containing a dissipation component $\mu_{1} \dot{\phi}_{i}(t)$, a delayed conservative term $k \sum_{j=1, j \neq i}^{N} \sin \left[\phi_{j}\left(t-\tau_{j i}\right)-\phi_{i}(t)\right]$, and a forcing part $\mu_{1} \omega_{i}$ [16]. Consequently, it is reasonable to suppose that the long-term solution of the system is a synchronous state, as shown in $[14,18]$, with the phases of all nodes oscillating with the same frequency $\omega_{s}$ that can be estimated. Thus, in order to estimate this frequency, the following hypotheses are considered [13]:

(a) $\dot{\phi}_{i}(t)=\omega_{s}$,

(b) $\ddot{\phi}_{i}(t)=0$,

(c) $\phi_{j}\left(t-\tau_{j i}\right) \approx \phi_{j}(t)+\dot{\phi}_{i}(t)\left(-\tau_{j i}\right) \approx \phi_{j}(t)-\omega_{s} \tau_{j i}$.

Therefore,

$$
\mu_{1}\left(\omega_{s}-\omega_{i}\right)-k\left[\sum_{j=1, j \neq i}^{N} \sin \left(\Delta \phi_{j i}-\omega_{s} \tau_{j i}\right)\right]=0 .
$$

The values of $\left(\Delta \phi_{j i}-\omega_{s} \tau_{j i}\right)$ are considered to be small because in the majority of the practical situations, the network is operating in the lock-in mode [17]. Consequently, (2.11) can be written as a linear approximation considering $\sin \left[\Delta \phi_{j i}-\omega_{s} \tau_{j i}\right] \approx \Delta \phi_{j i}-\omega_{s} \tau_{j i}$. Hence, for each node $i$,

$$
\mu_{1} \omega_{s}-\mu_{1} \omega_{i}-k\left[\sum_{j=1, j \neq i}^{N} \Delta \phi_{j i}\right]+k \omega_{s}\left[\sum_{j=1, j \neq i}^{N} \tau_{j i}\right]=0 .
$$

Using (2.12) for an $N$-node network, with $i, j=1, \ldots, N$, and $j \neq i$, as well as adding the $N$ resulting equations, as the sum of the terms $\Delta \phi_{j i}$ is equal to zero because $\Delta \phi_{j i}=-\Delta \phi_{i j}$, one can write

$$
N \mu_{1} \omega_{s}-\mu_{1} \sum_{i=1}^{N} \omega_{i}+k \omega_{s}\left(\sum_{i=1}^{N} \sum_{j=1, j \neq i}^{N} \tau_{j i}\right)=0 .
$$

Calculating $\omega_{s}$ from (2.13),

$$
\omega_{s}=\frac{\mu_{1}\left(\sum_{i=1}^{N} \omega_{i}\right)}{N \mu_{1}+k\left(\sum_{i=1}^{N} \sum_{j=i, j \neq i}^{N} \tau_{j i}\right)} .
$$

Dividing (2.14) by $N \mu_{1}$, and replacing $k=\mu_{1} \mu_{2} /(N-1)$, the estimation of the synchronous state frequency $\left(\omega_{s}\right)$ is obtained:

$$
\omega_{s}=\frac{(1 / N)\left(\sum_{i=1}^{N} \omega_{i}\right)}{1+\left(\mu_{2} / N(N-1)\right)\left(\sum_{i=1}^{N} \sum_{j=i, j \neq i}^{N} \tau_{j i}\right)} .
$$


Therefore, expression (2.15) is an estimation for the frequency of the synchronous state for a fully connected second-order PLL network, depending on the individual free-running frequencies and propagation delays. Notice that when the delays are zero, $\omega_{s}$ is given by the mean value of $\omega_{i}$. As it was considered that all free-running frequencies are different, there are phase shifts between the nodes in the synchronous state. In practical cases, as in communication networks, these phase differences are object of delay compensation techniques [5].

In previous work [13], numerical simulations were conducted to investigate the accuracy of expression (2.15) and to analyze how gains and delays change the behavior of the network. Here, the dynamic equations for the phase differences are derived allowing the analytic study of the local stability of the synchronous state.

\section{Phase difference equations}

In this section, the equations describing the dynamics of the phase errors, $\Delta \phi_{j i}(t)$, are derived. A set of $N-1$ second-order ordinary differential equations is obtained, expressing the phase differences between all the nodes and the node 1 , taken as reference.

Starting with the individual dynamical equations for each VCO phase and expressing the differences between the nodes $2,3, \ldots, N$ and node 1 , equations for $\Delta \phi_{j 1}(t), j=2,3, \ldots, N$ are written as below:

$$
\begin{aligned}
& \text { VCO 1: } \quad \ddot{\phi}_{1}(t)+\mu_{1} \dot{\phi}_{1}(t)-\mu_{1} \omega_{1}-k \\
& \times\left\{\sin \left[\phi_{2}\left(t-\tau_{21}\right)-\phi_{1}(t)\right]+\sin \left[\phi_{3}\left(t-\tau_{31}\right)-\phi_{1}(t)\right]\right. \\
& \left.+\sin \left[\phi_{4}\left(t-\tau_{41}\right)-\phi_{1}(t)\right]+\cdots+\sin \left[\phi_{N}\left(t-\tau_{N 1}\right)-\phi_{1}(t)\right]\right\}=0 ; \\
& \times\left\{\sin \left[\phi_{1}\left(t-\tau_{12}\right)-\phi_{2}(t)\right]+\sin \left[\phi_{3}\left(t-\tau_{32}\right)-\phi_{2}(t)\right]\right. \\
& \left.+\sin \left[\phi_{4}\left(t-\tau_{42}\right)-\phi_{2}(t)\right]+\cdots+\sin \left[\phi_{N}\left(t-\tau_{N 2}\right)-\phi_{2}(t)\right]\right\}=0 ; \\
& \times\left\{\sin \left[\phi_{1}\left(t-\tau_{13}\right)-\phi_{3}(t)\right]+\sin \left[\phi_{2}\left(t-\tau_{32}\right)-\phi_{3}(t)\right]\right. \\
& \left.+\sin \left[\phi_{4}\left(t-\tau_{43}\right)-\phi_{3}(t)\right]+\cdots+\sin \left[\phi_{N}\left(t-\tau_{N 3}\right)-\phi_{3}(t)\right]\right\}=0 ; \\
& \times\left\{\sin \left[\phi_{1}\left(t-\tau_{1 N-1}\right)-\phi_{N-1}(t)\right]+\sin \left[\phi_{2}\left(t-\tau_{2 N-1}\right)-\phi_{N-1}(t)\right]\right. \\
& \left.+\sin \left[\phi_{3}\left(t-\tau_{3 N-1}\right)-\phi_{N-1}(t)\right]+\cdots+\sin \left[\phi_{N}\left(t-\tau_{N-1 N}\right)-\phi_{N-1}(t)\right]\right\}=0 ; \\
& \times\left\{\sin \left[\phi_{1}\left(t-\tau_{1 N}\right)-\phi_{N}(t)\right]+\sin \left[\phi_{2}\left(t-\tau_{2 N}\right)-\phi_{N}(t)\right]\right. \\
& \left.+\sin \left[\phi_{3}\left(t-\tau_{3 N}\right)-\phi_{N}(t)\right]+\cdots+\sin \left[\phi_{N-1}\left(t-\tau_{N-1 N}\right)-\phi_{N}(t)\right]\right\}=0 .
\end{aligned}
$$


José R. C. Piqueira et al.

Using the condition $\phi_{i}\left(t-\tau_{j i}\right) \approx \phi_{i}(t)-\omega_{s} \tau_{j i}$ and considering $\Delta \phi_{j 1}(t)=\phi_{j}(t)-\phi_{1}(t)$, then

VCO 1: $\quad \ddot{\phi}_{1}(t)+\mu_{1} \dot{\phi}_{1}-\mu_{1} \omega_{1}-k$

$$
\begin{aligned}
& \times\left\{\sin \left[\Delta \phi_{21}(t)-\omega_{s} \tau_{21}\right]+\sin \left[\Delta \phi_{31}(t)-\omega_{s} \tau_{31}\right]\right. \\
& \left.\quad+\sin \left[\Delta \phi_{41}(t)-\omega_{s} \tau_{41}\right]+\cdots+\sin \left[\Delta \phi_{N 1}(t)-\omega_{s} \tau_{N 1}\right]\right\}=0 ;
\end{aligned}
$$

VCO $2: \quad \ddot{\phi}_{2}(t)+\mu_{1} \dot{\phi}_{2}(t)-\mu_{1} \omega_{2}-k$

$$
\begin{aligned}
& \times\left\{\sin \left[\Delta \phi_{12}(t)-\omega_{s} \tau_{12}\right]+\sin \left[\Delta \phi_{32}(t)-\omega_{s} \tau_{32}\right]\right. \\
& \left.\quad+\sin \left[\Delta \phi_{42}(t)-\omega_{s} \tau_{42}\right]+\cdots+\sin \left[\Delta \phi_{N 2}(t)-\omega_{s} \tau_{N 2}\right]\right\}=0 ;
\end{aligned}
$$

VCO 3: $\quad \ddot{\phi}_{3}(t)+\mu_{1} \dot{\phi}_{3}(t)-\mu_{1} \omega_{3}-k$

$$
\begin{aligned}
& \times\left\{\sin \left[\Delta \phi_{13}(t)-\omega_{s} \tau_{13}\right]+\sin \left[\Delta \phi_{23}(t)-\omega_{s} \tau_{23}\right]\right. \\
& \left.\quad+\sin \left[\Delta \phi_{43}(t)-\omega_{s} \tau_{43}\right]+\cdots+\sin \left[\Delta \phi_{N 3}(t)-\omega_{s} \tau_{N 3}\right]\right\}=0
\end{aligned}
$$

VCO $N-1: \quad \ddot{\phi}_{N-1}(t)+\mu_{1} \dot{\phi}_{N-1}(t)-\mu_{1} \omega_{N-1}-k$

$$
\begin{aligned}
& \times\left\{\sin \left[\Delta \phi_{1 N-1}(t)-\omega_{s} \tau_{1 N-1}\right]+\sin \left[\Delta \phi_{2 N-1}(t)-\omega_{s} \tau_{2 N-1}\right]\right. \\
& \left.\quad+\sin \left[\Delta \phi_{3 N-1}(t)-\omega_{s} \tau_{3 N-1}\right]+\cdots+\sin \left[\Delta \phi_{N N-1}(t)-\omega_{s} \tau_{N N-1}\right]\right\}=0 ;
\end{aligned}
$$

VCO $N: \quad \ddot{\phi}_{N}(t)+\mu_{1} \dot{\phi}_{N}(t)-\mu_{1} \omega_{N}-k$

$$
\begin{aligned}
& \times\left\{\sin \left[\Delta \phi_{1 N}(t)-\omega_{s} \tau_{1 N}\right]+\sin \left[\Delta \phi_{2 N}(t)-\omega_{s} \tau_{2 N}\right]\right. \\
& \left.\quad+\sin \left[\Delta \phi_{3 N}(t)-\omega_{s} \tau_{3 N}\right]+\cdots+\sin \left[\Delta \phi_{N-1 N}(t)-\omega_{s} \tau_{N-1 N}\right]\right\}=0 .
\end{aligned}
$$

Expressing the differences with node 1 as reference,

VCOs 1 and $2: \quad \ddot{\phi}_{2}(t)-\ddot{\phi}_{1}(t)+\mu_{1}\left(\dot{\phi}_{2}(t)-\dot{\phi}_{1}(t)\right)-\mu_{1}\left(\omega_{2}-\omega_{1}\right)$

$$
\begin{aligned}
& -k \sin \left[\Delta \phi_{12}(t)-\omega_{s} \tau_{12}\right]-k \sin \left[\Delta \phi_{32}(t)+\omega_{s} \tau_{32}\right] \\
& -k \sin \left[\Delta \phi_{42}(t)-\omega_{2} \tau_{42}\right]-\cdots-k \sin \left[\Delta \phi_{N 2}(t)-\omega_{s} \tau_{N 2}\right] \\
& +k \sin \left[\Delta \phi_{21}(t)-\omega_{s} \tau_{21}\right]+\cdots+k \sin \left[\Delta \phi_{N 1}(t)-\omega_{s} \tau_{N 1}\right]=0 ;
\end{aligned}
$$

VCOs 1 and 3: $\ddot{\phi}_{3}(t)-\ddot{\phi}_{1}(t)+\mu_{1}\left(\dot{\phi}_{3}(t)-\dot{\phi}_{1}(t)\right)-\mu_{1}\left(\omega_{3}-\omega_{1}\right)$

$$
\begin{aligned}
& -k \sin \left[\Delta \phi_{13}(t)-\omega_{s} \tau_{13}\right]-k \sin \left[\Delta \phi_{23}(t)-\omega_{s} \tau_{23}\right] \\
& -k \sin \left[\Delta \phi_{43}(t)-\omega_{2} \tau_{43}\right]-\cdots-k \sin \left[\Delta \phi_{N 3}(t)-\omega_{s} \tau_{N 3}\right] \\
& +k \sin \left[\Delta \phi_{21}(t)+\omega_{s} \tau_{21}\right]+k \sin \left[\Delta \phi_{31}(t)+\omega_{s} \tau_{31}\right] \\
& +k \sin \left[\Delta \phi_{41}(t)+\omega_{s} \tau_{41}\right]+\cdots+k \sin \left[\phi_{N 1}(t)-\omega_{s} \tau_{N 1}\right]=0 ;
\end{aligned}
$$

$\vdots$ 
VCOs 1 and $N: \quad \ddot{\phi}_{N}(t)-\ddot{\phi}_{1}(t)+\mu_{1}\left(\dot{\phi}_{N}(t)-\dot{\phi}_{1}(t)\right)-\mu_{1}\left(\omega_{N}-\omega_{1}\right)$

$$
\begin{aligned}
& -k \sin \left[\Delta \phi_{1 N}(t)-\omega_{s} \tau_{1 N}\right]-k \sin \left[\Delta \phi_{2 N}(t)-\omega_{s} \tau_{2 N}\right] \\
& -k \sin \left[\Delta \phi_{3 N}(t)-\omega_{2} \tau_{3 N}\right]-\cdots-k \sin \left[\Delta \phi_{N-1 N}(t)-\omega_{s} \tau_{N-1 N}\right] \\
& +k \sin \left[\Delta \phi_{21}(t)+\omega_{s} \tau_{21}\right]+k \sin \left[\Delta \phi_{31}(t)+\omega_{s} \tau_{31}\right] \\
& +k \sin \left[\Delta \phi_{41}(t)+\omega_{s} \tau_{52}\right]+\cdots+k \sin \left[\phi_{N 1}(t)-\omega_{s} \tau_{N 1}\right]=0 .
\end{aligned}
$$

Replacing the terms $\Delta \phi_{j 1}(t)=\phi_{j}(t)-\phi_{1}(t), \Delta \dot{\phi}_{j 1}(t)=\dot{\phi}_{j}(t)-\dot{\phi}_{1}(t), \Delta \ddot{\phi}_{j 1}(t)=\ddot{\phi}_{j}(t)-\ddot{\phi}_{1}(t)$, and using the identity $\sin \left[\Delta \phi_{j 1}(t)-\omega_{s} \tau_{j 1}\right]+\sin \left[\Delta \phi_{j i}(t)+\omega_{s} \tau_{j i}\right]=2 \sin \Delta \phi_{j i} \cos \omega_{s} \tau_{j i}$ in the former expressions, the VCO phase differences become

VCOs 1 and 2: $\quad \Delta \ddot{\phi}_{21}(t)+\mu_{1} \Delta \dot{\phi}_{21}(t)-\mu_{1} \Delta \omega_{21}+2 k \sin \Delta \phi_{21}(t) \cos \left(\omega_{s} \tau_{21}\right)$

$$
\begin{aligned}
& -k \sin \left[\Delta \phi_{32}(t)+\omega_{s} \tau_{32}\right]-k \sin \left[\Delta \phi_{42}(t)-\omega_{2} \tau_{42}\right] \\
& -\cdots-k \sin \left[\Delta \phi_{N 2}(t)-\omega_{s} \tau_{N 2}\right]+k \sin \left[\Delta \phi_{31}(t)-\omega_{s} \tau_{31}\right] \\
& +k \sin \left[\Delta \phi_{41}(t)-\omega_{s} \tau_{41}\right]+\cdots+k \sin \left[\Delta \phi_{N 1}(t)-\omega_{s} \tau_{N 1}\right]=0 ;
\end{aligned}
$$

VCOs 1 and 3: $\quad \Delta \ddot{\phi}_{31}(t)+\mu_{1} \Delta \dot{\phi}_{31}(t)-\mu_{1} \Delta \omega_{31}+2 k \sin \Delta \phi_{31}(t) \cos \left(\omega_{s} \tau_{31}\right)$

$$
\begin{aligned}
& -k \sin \left[\Delta \phi_{23}(t)+\omega_{s} \tau_{23}\right]-k \sin \left[\Delta \phi_{43}(t)-\omega_{2} \tau_{43}\right] \\
& -\cdots-k \sin \left[\Delta \phi_{N 3}(t)-\omega_{s} \tau_{N 3}\right]+k \sin \left[\Delta \phi_{21}(t)-\omega_{s} \tau_{21}\right] \\
& +k \sin \left[\Delta \phi_{31}(t)-\omega_{s} \tau_{31}\right]+\cdots+k \sin \left[\Delta \phi_{N 1}(t)-\omega_{s} \tau_{N 1}\right]=0 ;
\end{aligned}
$$

VCOs 1 and $N: \quad \Delta \ddot{\phi}_{N 1}(t)+\mu_{1} \Delta \dot{\phi}_{N 1}(t)-\mu_{1} \Delta \omega_{N 1}+2 k \sin \Delta \phi_{N 1}(t) \cos \left(\omega_{s} \tau_{N 1}\right)$

$$
\begin{aligned}
& -k \sin \left[\Delta \phi_{2 N}(t)+\omega_{s} \tau_{2 N}\right]-k \sin \left[\Delta \phi_{3 N}(t)-\omega_{2} \tau_{3 N}\right] \\
& -\cdots-k \sin \left[\Delta \phi_{N-1 N}(t)-\omega_{s} \tau_{N-1 N}\right]+k \sin \left[\Delta \phi_{21}(t)-\omega_{s} \tau_{21}\right] \\
& +k \sin \left[\Delta \phi_{31}(t)-\omega_{s} \tau_{31}\right]+\cdots+k \sin \left[\Delta \phi_{N-11}(t)-\omega_{s} \tau_{N-11}\right]=0 .
\end{aligned}
$$

These equations give the general expression that describes the dynamical behavior of the phase differences for the network VCOs as below:

$$
\begin{aligned}
& \Delta \ddot{\phi}_{j 1}(t)+\mu_{1} \Delta \dot{\phi}_{j 1}(t)-\mu_{1} \Delta \omega_{j 1}+2 k \sin \Delta \phi_{j 1}(t) \cos \left(\omega_{s} \tau_{j 1}\right) \\
& \quad-k\left\{\sum_{m=2, m \neq j}^{N} \sin \left[\Delta \phi_{m j}(t)-\omega_{s} \tau_{m j}\right]\right\}+k\left\{\sum_{m=2, m \neq j}^{N} \sin \left[\Delta \phi_{m 1}(t)-\omega_{s} \tau_{m 1}\right]\right\}=0 .
\end{aligned}
$$


The nonlinear differential equation (3.5), for small-phase deviations, can be approximately expressed by the linear term of the Taylor series expansion [16]. Then, rewriting (3.5)

$$
\begin{aligned}
& \Delta \ddot{\phi}_{j 1}(t)+\mu_{1} \Delta \dot{\phi}_{j 1}(t)-\mu_{1} \Delta \omega_{j 1}+2 k \Delta \phi_{j 1}(t) \\
& \quad-k\left\{\sum_{m=2, m \neq j}^{N}\left[\Delta \phi_{m j}(t)-\Delta \phi_{m 1}(t)\right]\right\}+k \omega_{s}\left\{\sum_{m=1, m \neq j}^{N}\left[\tau_{m j}-\tau_{m 1}\right]\right\}=0 .
\end{aligned}
$$

Expressions (3.5) and (3.6) describe the dynamics of the phase adjustments of a fully connected PLL network depending on the PLL node parameters $\mu_{1}$ and $k$, the number of nodes $N$, as well as the individual free-running frequencies and the delays. These equations allow the research of the synchronous state stability that is conducted in the next section.

\section{Synchronous state stability}

In Section 2, an expression for the synchronous state frequency for the fully connected network was derived and, in this section, the stability of the synchronous state is studied, that is, if the reachable synchronous state is robust under small perturbations. The analysis is performed considering that the solutions of the linear equation (3.6) can be topologically equivalent to the solutions of (3.5) in a small neighborhood of the synchronous state if it is a hyperbolic equilibrium point [16]. The procedure will be shown for three-node and four-node networks providing the identification of patterns in the expressions for the eigenvalues of the Jacobian matrix representing the linear equivalent system around the synchronous state.

\subsection{Three-node network}

For a three-node network, the phase differences are $\Delta \phi_{12}$ and $\Delta \phi_{13}$. By using (3.5), the system is described by

VCOs 1 and $2: \quad \Delta \ddot{\phi}_{12}+\mu_{1} \Delta \dot{\phi}_{12}+2 k \Delta \phi_{12}+k \Delta \phi_{13}-k \Delta \phi_{23}+\underbrace{\mu_{1} \Delta \omega_{21}+k \omega_{s}\left(\tau_{13}-\tau_{23}\right)}_{a}=0$
VCOs 1 and 3: $\Delta \ddot{\phi}_{13}+\mu_{1} \Delta \dot{\phi}_{13}+2 k \Delta \phi_{13}+k \Delta \phi_{12}+k \Delta \phi_{23}+\underbrace{\mu_{1} \Delta \omega_{31}+k \omega_{s}\left(\tau_{12}-\tau_{32}\right)}_{b}=0$.

The system composed of two second-order equations (4.1) can be described by the state variables:

$$
\begin{array}{ll}
x_{1} \equiv \Delta \phi_{12}, & x_{3} \equiv \Delta \phi_{13}, \\
x_{2} \equiv \Delta \dot{\phi}_{12}, & x_{4} \equiv \Delta \dot{\phi}_{13} .
\end{array}
$$

Considering that $\Delta \phi_{23}=x_{3}-x_{1}$, the following first-order equations result:

$$
\begin{aligned}
& \dot{x}_{1}=x_{2}, \\
& \dot{x}_{2}=-3 k x_{1}-\mu_{1} x_{2}-a, \\
& \dot{x}_{3}=x_{4}, \\
& \dot{x}_{4}=-3 k x_{3}-\mu_{1} x_{4}-b .
\end{aligned}
$$


Consequently, the Jacobian matrix $J$ from (4.3) is

$$
J=\left[\begin{array}{cccc}
0 & 1 & 0 & 0 \\
-3 k & -\mu_{1} & 0 & 0 \\
0 & 0 & 0 & 1 \\
0 & 0 & -3 k & -\mu_{1}
\end{array}\right]
$$

The eigenvalues of $J$ are the roots of the characteristic polynomial

$$
\left(\lambda^{2}+\mu_{1} \lambda+3 k\right)^{2}=0
$$

which have multiplicity two, and are given by

$$
\begin{aligned}
& \lambda_{1}=-\frac{\mu_{1}}{2}+\frac{\sqrt{\mu_{1}^{2}-12 k}}{2}, \\
& \lambda_{2}=-\frac{\mu_{1}}{2}-\frac{\sqrt{\mu_{1}^{2}-12 k}}{2} .
\end{aligned}
$$

\subsection{Four-node network}

The calculation of the eigenvalues for the four-node network follows the same procedure of the former case. The phase differences are represented by $\Delta \phi_{12}, \Delta \phi_{13}$, and $\Delta \phi_{14}$. By using (3.5), the system is written as

VCOs 1 and $2: \quad \Delta \ddot{\phi}_{12}+\mu_{1} \Delta \dot{\phi}_{12}+2 k \Delta \phi_{12}+k \Delta \phi_{13}-k \Delta \phi_{23}+k \Delta \phi_{14}-k \Delta \phi_{24}$

$$
+\underbrace{\mu_{1} \Delta \omega_{21}+k \omega_{s}\left(\tau_{13}-\tau_{23}+\tau_{14}-\tau_{24}\right)}_{a}=0
$$

VCOs 1 and 3: $\quad \Delta \ddot{\phi}_{13}+\mu_{1} \Delta \dot{\phi}_{13}+2 k \Delta \phi_{13}+k \Delta \phi_{12}+k \Delta \phi_{23}+k \Delta \phi_{14}-k \Delta \phi_{34}$

$$
+\underbrace{\mu_{1} \Delta \omega_{31}+k \omega_{s}\left(\tau_{12}-\tau_{32}+\tau_{14}-\tau_{34}\right)}_{b}=0
$$

VCOs 1 and $4: \quad \Delta \ddot{\phi}_{14}+\mu_{1} \Delta \dot{\phi}_{14}+2 k \Delta \phi_{14}+k \Delta \phi_{12}+k \Delta \phi_{24}+k \Delta \phi_{13}+k \Delta \phi_{34}$

$$
+\underbrace{\mu_{1} \Delta \omega_{41}+k \omega_{s}\left(\tau_{12}-\tau_{42}+\tau_{13}-\tau_{43}\right)}_{c}=0
$$

The second-order expressions (4.7) can be rewritten in terms of state variables:

$$
\begin{aligned}
& x_{1} \equiv \Delta \phi_{12}, \quad x_{3} \equiv \Delta \phi_{13}, \quad x_{5} \equiv \Delta \phi_{14}, \\
& x_{2} \equiv \Delta \dot{\phi}_{12}, \quad x_{4} \equiv \Delta \dot{\phi}_{13}, \quad x_{6} \equiv \Delta \dot{\phi}_{14} \text {. }
\end{aligned}
$$


José R. C. Piqueira et al.

Considering that $\Delta \phi_{23}=x_{3}-x_{1}, \Delta \phi_{24}=x_{5}-x_{1}$, and $\Delta \phi_{34}=x_{3}-x_{5}$, the following first-order equations result:

$$
\begin{aligned}
& \dot{x}_{1}=x_{2}, \\
& \dot{x}_{2}=-4 k x_{1}-\mu_{1} x_{2}-a, \\
& \dot{x}_{3}=x_{4}, \\
& \dot{x}_{4}=-4 k x_{3}-\mu_{1} x_{4}-b, \\
& \dot{x}_{5}=x_{6}, \\
& \dot{x}_{6}=-4 k x_{5}-\mu_{1} x_{6}-c .
\end{aligned}
$$

Consequently, the Jacobian matrix $J$ from (4.9) is

$$
J=\left[\begin{array}{cccccc}
0 & 1 & 0 & 0 & 0 & 0 \\
-4 k & -\mu_{1} & 0 & 0 & 0 & 0 \\
0 & 0 & 0 & 1 & 0 & 0 \\
0 & 0 & -4 k & -\mu_{1} & 0 & 0 \\
0 & 0 & 0 & 0 & 0 & 1 \\
0 & 0 & 0 & 0 & -4 k & -\mu_{1}
\end{array}\right]
$$

The eigenvalues of $J$ are given by the roots of the characteristic polynomial:

$$
\left(\lambda^{2}+\mu_{1} \lambda+4 k\right)^{3}=0
$$

which have multiplicity 3 , and are

$$
\begin{aligned}
& \lambda_{1}=-\frac{\mu_{1}}{2}+\frac{\sqrt{\mu_{1}^{2}-16 k}}{2}, \\
& \lambda_{2}=-\frac{\mu_{1}}{2}-\frac{\sqrt{\mu_{1}^{2}-16 k}}{2} .
\end{aligned}
$$

\section{3. $N$-node network}

As it was shown, for a fully connected PLL network, the Taylor series development around the synchronous state results in Jacobian matrices with a canonical Jordan form [24]. Table 1 shows the characteristic polynomials and the expressions of the eigenvalues and their multiplicities, $\mathrm{m}$, corresponding to fully connected networks dynamic equations, around the synchronous state, for different number, $n$, of nodes.

It can be observed that for any number of nodes, the eigenvalues from Table 1 are with negative real parts, implying that the synchronous state is locally asymptotically stable. 
Table 1: Eigenvalues for an $N$-node network.

\begin{tabular}{cccccc}
\hline$n$ & & \multicolumn{2}{c}{ Eigenvalues and multiplicities } & \\
\hline 2 & $\left(\lambda^{2}+\mu_{1} \lambda+2 k\right)$ & $-\frac{\mu_{1}}{2}+\frac{\sqrt{\mu_{1}^{2}-8 k}}{2}$ & 1 & $-\frac{\mu_{1}}{2}-\frac{\sqrt{\mu_{1}^{2}-8 k}}{2}$ & 1 \\
3 & $\left(\lambda^{2}+\mu_{1} \lambda+3 k\right)^{2}$ & $-\frac{\mu_{1}}{2}+\frac{\sqrt{\mu_{1}^{2}-12 k}}{2}$ & 2 & $-\frac{\mu_{1}}{2}-\frac{\sqrt{\mu_{1}^{2}-12 k}}{2}$ & 2 \\
4 & $\left(\lambda^{2}+\mu_{1} \lambda+4 k\right)^{3}$ & $-\frac{\mu_{1}}{2}+\frac{\sqrt{\mu_{1}^{2}-16 k}}{2}$ & 3 & $-\frac{\mu_{1}}{2}-\frac{\sqrt{\mu_{1}^{2}-16 k}}{2}$ & 3 \\
5 & $\left(\lambda^{2}+\mu_{1} \lambda+5 k\right)^{4}$ & $-\frac{\mu_{1}}{2}+\frac{\sqrt{\mu_{1}^{2}-20 k}}{2}$ & 4 & $-\frac{\mu_{1}}{2}-\frac{\sqrt{\mu_{1}^{2}-20 k}}{2}$ & 4 \\
6 & $\left(\lambda^{2}+\mu_{1} \lambda+6 k\right)^{5}$ & $-\frac{\mu_{1}}{2}+\frac{\sqrt{\mu_{1}^{2}-246 k}}{2}$ & 5 & $-\frac{\mu_{1}}{2}-\frac{\sqrt{\mu_{1}^{2}-24 k}}{2}$ & 5 \\
7 & $\left(\lambda^{2}+\mu_{1} \lambda+7 k\right)^{6}$ & $-\frac{\mu_{1}}{2}+\frac{\sqrt{\mu_{1}^{2}-28 k}}{2}$ & 6 & $-\frac{\mu_{1}}{2}-\frac{\sqrt{\mu_{1}^{2}-28 k}}{2}$ & 6 \\
$\vdots$ & $\vdots$ & $\vdots$ & $\vdots$ & $-\frac{\mu_{1}}{2}-\frac{\sqrt{\mu_{1}^{2}-4 N k}}{2}$ & $(N-1)$ \\
$N$ & $\left(\lambda^{2}+\mu_{1} \lambda+N k\right)^{(N-1)}$ & $-\frac{\mu_{1}}{2}+\frac{\sqrt{\mu_{1}^{2}-4 N k}}{2}$ & $(N-1)$ &
\end{tabular}

\section{Conclusions}

As the fully connected architecture started to be used in large scale in clock distribution systems, (2.15) can be applied by network designers as an estimation for the frequency of the synchronous state when second-order PLLs are used to extract the timing information in the nodes.

Besides, because the synchronous state is a hyperbolic locally asymptotically stable equilibrium point, the network can recover synchronization after perturbation. The eigenvalues presented in Table 1 can be used for determining the transient behavior of such a resynchronization process.

\section{Acknowledgment}

José R. C. Piqueira is supported by FAPESP and CNPq. Luiz H. A. Monteiro is partially supported by $\mathrm{CNPq}$

\section{References}

[1] S. Bregni, Synchronization of Digital Networks, John Wiley \& Sons, Chichester, UK, 1st edition, 2002.

[2] H. A. Tanaka, A. Hasegawa, H. Mizuno, and T. Endo, "Synchronizability of distributed clock oscillators," IEEE Transactions on Circuits and Systems I, vol. 49, no. 9, pp. 1271-1278, 2002.

[3] M.-H. Hung, J. Tsai, F.-T. Cheng, and H.-C. Yang, "Development of an Ethernet-based equipment integration framework for factory automation," Robotics and Computer-Integrated Manufacturing, vol. 20, no. 5, pp. 369-383, 2004. 
José R. C. Piqueira et al.

[4] P. Kellett, "Beyond the LAN: Ethernet's evolution into the public network," Fiber and Integrated Optics, vol. 22, no. 5, pp. 289-303, 2003.

[5] W. C. Lindsey, F. Ghazvinian, W. C. Hagmann, and K. Dessouky, "Network synchronization," Proceedings of the IEEE, vol. 73, no. 10, pp. 1445-1467, 1985.

[6] D. J. Messerschmitt, "Synchronization in digital system design," IEEE Journal on Selected Areas in Communications, vol. 8, no. 8, pp. 1404-1419, 1990.

[7] M. Saint-Laurent and M. Swaminathan, "A multi-PLL clock distribution architecture for gigascale integration," in Proceedings of the IEEE Computer Society Workshop on VLSI, pp. 30-35, Orlando, Fla, USA, May 2001.

[8] V. Gutnik and A. P. Chandrakasan, "Active GHz clock network using distributed PLLs," IEEE Journal of Solid-State Circuits, vol. 35, no. 11, pp. 1553-1560, 2000.

[9] M. Saint-Laurent, P. Zarkesh-Ha, M. Swaminathan, and J. D. Meindl, "Optimal clock distribution with an array of phase-locked loops for multiprocessor chips," in Proceedings of the 44th IEEE Midwest Symposium on Circuits and Systems (MWSCAS '01), vol. 1, pp. 454-457, Dayton, Ohio, USA, August 2001.

[10] M. M. Nordman, W. E. Kozlowski, and O. Vahamaki, "A method for synchronizing low cost energy aware sensors used in industrial process monitoring," in Proceedings of the 27th Annual Conference of the IEEE Industrial Electronics Society (IECON '01), vol. 1, pp. 100-106, Denver, Colo, USA, NovemberDecember 2001.

[11] F. C. Hoppensteadt and E. Izhikevich, "Pattern recognition via synchronization in phase-locked loop neural networks," IEEE Transactions on Neural Networks, vol. 11, no. 3, pp. 734-738, 2000.

[12] J. R. C. Piqueira, F. M. Orsatti, and L. H. A. Monteiro, "Computing with phase locked loops: choosing gains and delays," IEEE Transactions on Neural Networks, vol. 14, no. 1, pp. 243-247, 2003.

[13] J. R. C. Piqueira, M. Q. de Oliveira, and L. H. A. Monteiro, "Synchronous state in a fully connected phase-locked loop network," Mathematical Problems in Engineering, vol. 2006, Article ID 52356, 12 pages, 2006.

[14] L. H. A. Monteiro, N. C. F. Canto, J. G. Chaui-Berlinck, F. M. Orsatti, and J. R. C. Piqueira, “Global and partial synchronism in phase-locked loop networks," IEEE Transactions on Neural Networks, vol. 14, no. 6, pp. 1572-1575, 2003.

[15] J. R. C. Piqueira and L. H. A. Monteiro, "All-pole phase-locked loops: calculating lock-in range by using Evan's root-locus," International Journal of Control, vol. 79, no. 7, pp. 822-829, 2006.

[16] J. Guckenheimer and P. Holmes, Nonlinear Oscillations, Dynamical Systems, and Bifurcations of Vector Fields, vol. 42 of Applied Mathematical Sciences, Springer, New York, NY, USA, 2nd edition, 1983.

[17] R. E. Best, Phase-Locked Loops, McGraw Hill, New York, NY, USA, 4th edition, 1999.

[18] J. R. C. Piqueira, E. Y. Takada, and L. H. A. Monteiro, "Analyzing the effect of the phase-jitter in the operation of second order phase-locked loops," IEEE Transactions on Circuits and Systems II, vol. 52, no. 6, pp. 331-335, 2005.

[19] J. R. C. Piqueira, A. Z. Caligares, and L. H. A. Monteiro, "Double-frequency jitter figures in masterslave PLL networks," AEU - International Journal of Electronics and Communications, vol. 61, no. 10, pp. 678-683, 2007.

[20] J. R. C. Piqueira and L. H. A. Monteiro, "Considering second-harmonic terms in the operation of the phase detector for second order phase-locked loop," IEEE Transactions on Circuits and Systems I, vol. 50, no. 6, pp. 805-809, 2003.

[21] R. B. Pinheiro, J. J. da Cruz, and J. R. C. Piqueira, "Robust clock generation system," International Journal of Control, vol. 80, no. 1, pp. 35-44, 2007.

[22] F. M. Orsatti and J. R. C. Piqueira, "Evolutionary multi-objective optimization in mutually connected PLL synchronization networks. Modeling and control of autonomous decision support based systems," in Proceedings of the 13rd International Workshop on Dynamics and Control, vol. 1, pp. 255-262, Shaker, Wiesensteig, Germany, May 2005.

[23] L. H. A. Monteiro, D. N. F. Filho, and J. R. C. Piqueira, "Bifurcation analysis for third-order phaselocked loops," IEEE Signal Processing Letters, vol. 11, no. 5, pp. 494-496, 2004.

[24] M. W. Hirsch, S. Smale, and R. L. Devaney, Differential Equations, Dynamical Systems, \& An Introduction to Chaos, vol. 60 of Pure and Applied Mathematics, Elsevier/Academic Press, Amsterdam, The Netherlands, 2nd edition, 2004. 


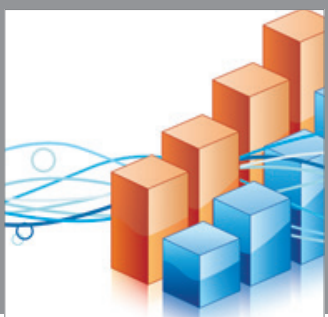

Advances in

Operations Research

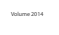

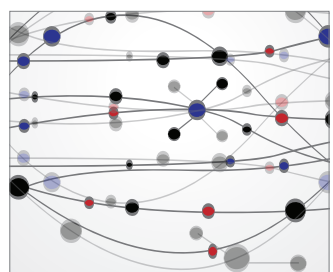

\section{The Scientific} World Journal
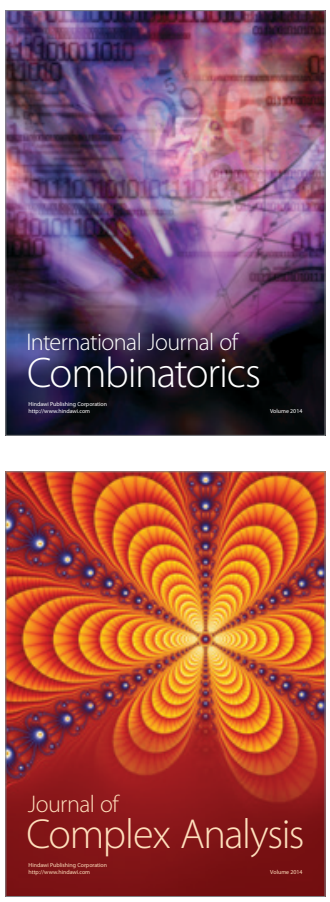

International Journal of

Mathematics and

Mathematical

Sciences
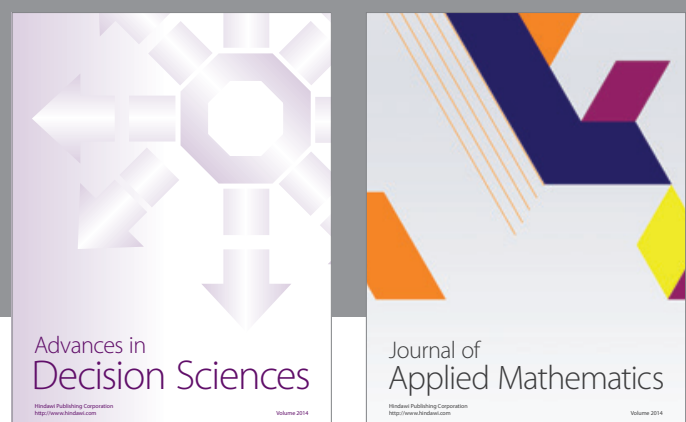

Journal of

Applied Mathematics
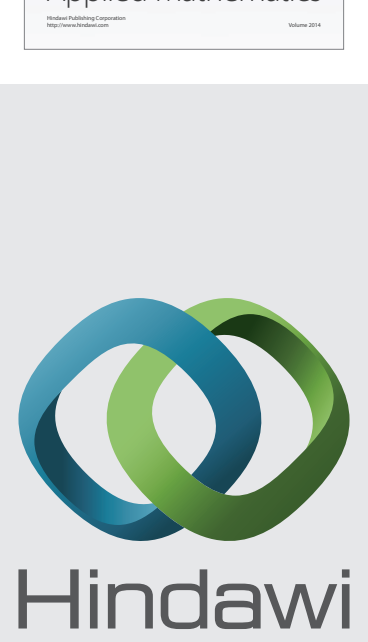

Submit your manuscripts at http://www.hindawi.com
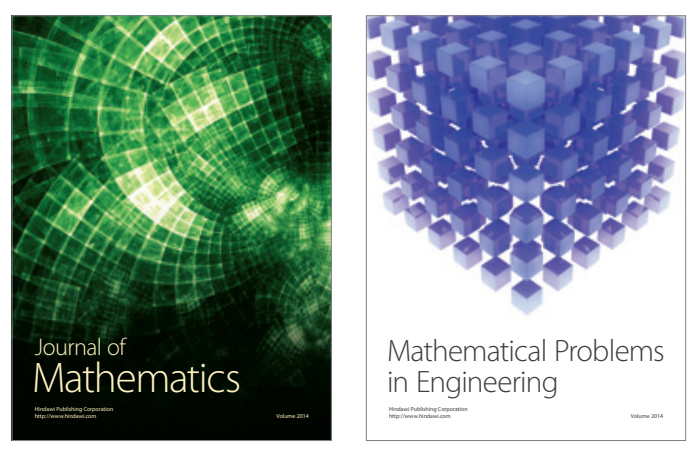

Mathematical Problems in Engineering
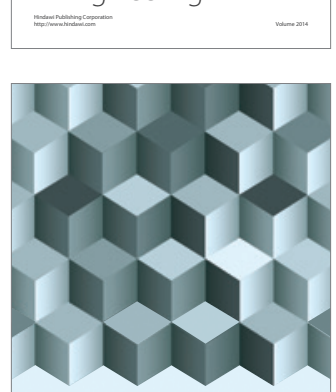

Journal of

Function Spaces
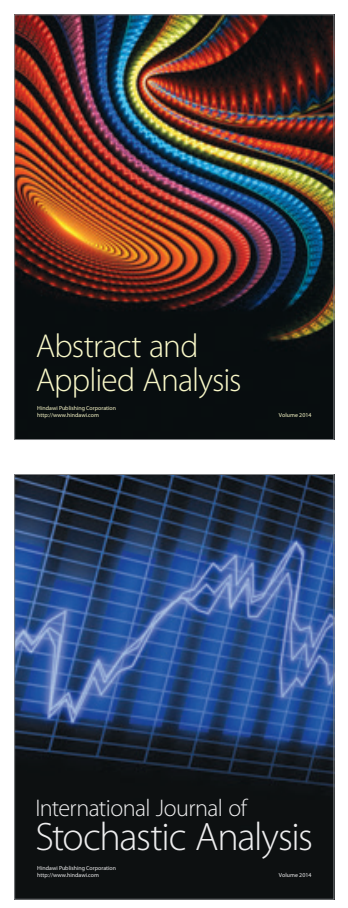

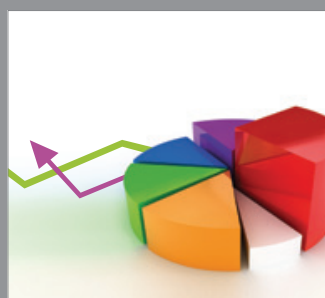

ournal of

Probability and Statistics

Promensencen
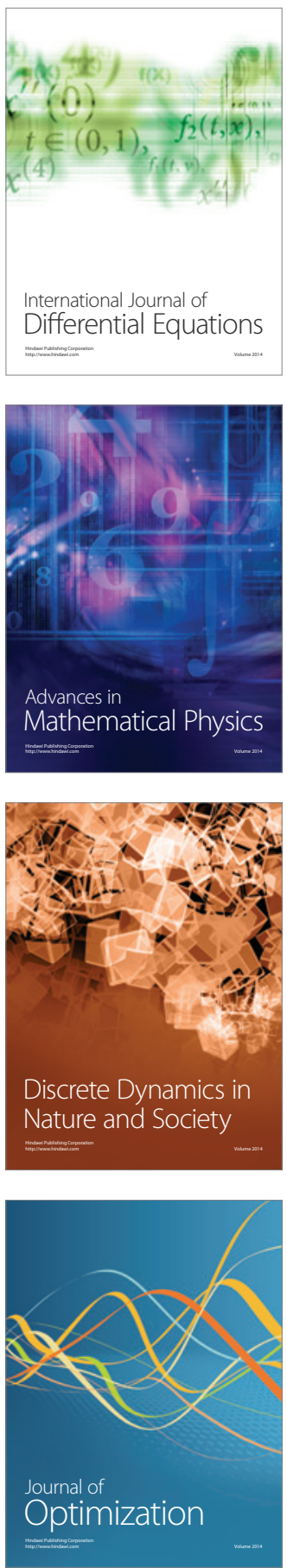